Pacific Journal of Mathematics

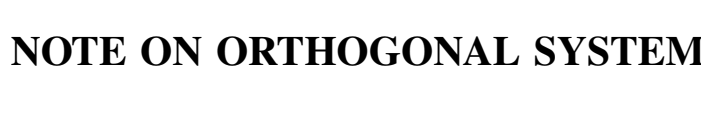




\section{A NOTE ON ORTHOGONAL SYSTEMS}

I. I. Hirschman, JR.

1. Let $\omega_{n}(x), n=1,2, \cdots, 0 \leqq x \leqq 1$, be an orthonormal set of functions which are uniformly bounded,

$$
\left|\omega_{n}(x)\right| \leqq M \quad(n=1,2, \cdots, 0 \leqq x \leqq 1) .
$$

If $\sum_{1}^{\infty}\left|a_{n}\right|<\infty$, and if $\int_{0}^{1}|g(x)| d x<\infty$ we may define

$$
f(x)=\sum_{n=1}^{\infty} a_{n} \omega_{n}(x), \quad b_{n}=\int_{0}^{1} g(x) \omega_{n}(x) d x .
$$

The following inequalities were established by R. E. A. C. Paley [1]:

$$
\begin{array}{ll}
{\left[\int_{0}^{1}|f(x)|^{q} d x\right]^{1 / q} \leqq A_{1}^{\prime}(q)\left[\sum_{n=1}^{\infty}\left|a_{n}\right|^{q} n^{q-2}\right]^{1 / q}} & (2 \leqq q<\infty) ; \\
{\left[\sum_{n=1}^{\infty}\left|b_{n}\right|^{p} n^{p-2}\right]^{1 / p} \leqq A_{2}^{\prime}(p)\left[\int_{0}^{1}|g(x)|^{p} d x\right]^{1 / p}} & (1<p \leqq 2) ; \\
{\left[\int_{0}^{1}|f(x)|^{p} x^{p-2} d x\right]^{1 / p} \leqq A_{3}^{\prime}(p)\left[\sum_{n=1}^{\infty}\left|a_{n}\right|^{p}\right]^{1 / p}} & (1<p \leqq 2) ; \\
{\left[\sum_{n=1}^{\infty}\left|b_{n}\right|^{q}\right]^{1 / q} \leqq A_{4}^{\prime}(q)\left[\int_{0}^{1}|g(x)|^{q} x^{q-2} d x\right]^{1 / q}} & (2 \leqq q<\infty) .
\end{array}
$$

In the present paper we shall establish some related results which are however a great deal simpler. We shall prove that

$$
\begin{array}{ll}
{\left[\int_{0}^{1}|f(x)|^{2} x^{-2 \alpha} d x\right]^{1 / 2} \leqq A_{1}(\alpha)\left[\sum_{n=1}^{\infty}\left|a_{n}\right|^{2} n^{2 \alpha}\right]^{1 / 2}} & (0 \leqq \alpha<1 / 2) ; \\
{\left[\sum_{n=1}^{\infty}\left|b_{n}\right|^{2} n^{-2 \alpha}\right]^{1 / 2} \leqq A_{2}(\alpha)\left[\int_{0}^{1}|g(x)|^{2} x^{2 \alpha} d x\right]^{1 / 2}} & (0 \leqq \alpha<1 / 2) .
\end{array}
$$

As Paley pointed out, the inequalities (3) include the inequalities of F. Riesz which assert that

$$
\begin{aligned}
& {\left[\int_{0}^{1}|f(x)|^{q} d x\right]^{1 / q} \leqq B(p)\left[\sum_{n=1}^{\infty}\left|a_{n}\right|^{p}\right]^{1 / p},} \\
& {\left[\sum_{n=1}^{\infty}\left|b_{n}\right|^{q}\right]^{1 / q} \leqq B(p)\left[\int_{0}^{1}|g(x)|^{p} d x\right]^{1 / p} \quad(1 \leqq p \leqq 2,1 / p+1 / q=1) .}
\end{aligned}
$$

Received December 2, 1954. Research supported in part by the Office of Ordnance Research under contract DA-23-072-ORD-392. 
(The best values of the constants $B(p)$ cannot be obtained by this argument however). The inequalities $\left(4^{\prime}\right)$ and $\left(4^{\prime \prime}\right)$ also include (5) (again not with the best values for $B(p)$ ). The demonstration of Riesz's theorem which one obtains in this way is unusually simple.

2. We now proceed to the demonstration of the inequalities $1(4)$. We assert that $A_{1}\left(\alpha^{\prime}\right)=A_{2}(\alpha)$ and thus that either of the inequalities implies the other. Suppose that the inequality $1\left(4^{\prime}\right)$ holds. We define

$$
F_{N}(x)=\sum_{n=1}^{N} b_{n} n^{-2 \alpha} \omega_{n}(x) \text {. }
$$

By assumption

$$
\int_{0}^{1}\left|F_{N}(x)\right|^{2} x^{-2 \alpha} d x \leqq A_{1}^{2}(\alpha) \sum_{n=1}^{N}\left|b_{n} n^{-2 \alpha}\right|{ }^{2} n^{2 \alpha}=A_{1}^{2}(\alpha) \sum_{n=1}^{N}\left|b_{n}\right|^{2} n^{-2 \alpha} .
$$

We have

$$
\begin{aligned}
& \sum_{n=1}^{N}\left|b_{n}\right|^{2} n^{-2 \alpha}=\int_{0}^{1} F_{N}(x) g(x) d x \leqq\left[\int_{0}^{1}\left|F_{N}(x)\right|^{2} x^{2 \alpha} d x\right]^{1 / 2}\left[\int_{0}^{1}|g(x)|^{2} x^{-2 \alpha} d x\right]^{1 / 2} \\
& \leqq \\
&\left.A_{1}^{2}(\alpha) \sum_{n=1}^{N}\left|b_{n}\right|^{2} n^{-2 \alpha}\right]^{1 / 2}\left[\int_{0}^{1}|g(x)|^{2} x^{2 \alpha} d x\right]^{1 / 2}, \\
& {\left[\sum_{n=1}^{N}\left|b_{n}\right|^{2} n^{-2 \alpha}\right]^{1 / 2} \leqq A_{1}(\alpha)\left[\int_{0}^{1}|g(x)|^{2} x^{2 \alpha} d x\right]^{1 / 2} . }
\end{aligned}
$$

Allowing $N$ to increase without limit we see that $A_{2}(\alpha) \leqq A_{1}(\alpha)$. Suppose now that $1\left(4^{\prime \prime}\right)$ holds. Set

$$
b_{n}=\int_{0}^{1} f(x) x^{-2 x} \overline{\omega_{n}(x)} d x .
$$

By assumption

$$
\sum_{n=1}^{\infty}\left|b_{n}\right|^{2} n^{-2 \alpha} \leqq A_{2}^{2}(\alpha) \int_{0}^{1}\left|f(x) x^{-2 \alpha}\right|^{2} x^{2 \alpha} d x=A_{2}^{2}(\alpha) \int_{0}^{1}|f(x)|^{2} x^{-2 \alpha} d x
$$

We have

$$
\begin{aligned}
& \int_{0}^{1}|f(x)|^{2} x^{-\Sigma \alpha} d x=\int_{L}^{1}\left[\sum_{n=1}^{\infty} a_{n} \omega_{n}(x)\right] f(x) x^{-2 \alpha} d x=\sum_{n=1}^{\infty} a_{n} \int_{0}^{1} f(x) x^{-2 \alpha} \omega_{n}(x) d x \\
& =\sum_{n=1}^{\infty} a_{n} \bar{b}_{n} \leqq\left[\sum_{n=1}^{N}\left|a_{n}\right|^{2} n^{2 \alpha}\right]^{1 / 2}\left[\sum_{n=1}^{\infty}\left|b_{n}\right|^{2} n^{-2 \alpha}\right]^{1 / 2} \\
& \leqq\left[A_{2}^{2}(\alpha) \int_{0}^{1}|f(x)|^{2} x^{-2 \alpha} d x\right]^{1 / 2}\left[\sum_{n=1}^{\infty}\left|a_{n}\right|^{2} n^{2 \alpha}\right]^{1 / 2}, \\
& {\left[\int_{0}^{1}|f(x)|^{2} x^{-2 \alpha} d x\right]^{1 / 2} \leqq A_{2}(\alpha)\left[\sum_{n=1}^{\infty}\left|a_{n}\right|^{2} n^{2 \alpha}\right]^{-1 / 2} \text {, }}
\end{aligned}
$$

and thus $A_{1}(\alpha) \leqq A_{2}(\alpha)$. Since $A_{1}(\alpha)=A_{2}(\alpha)$ we may write $A(\alpha)$ for $A_{1}(\alpha)$ 
and $A_{2}(\alpha)$.

It is evidently sufficient to prove either $1\left(4^{\prime}\right)$ or $1\left(4^{\prime \prime}\right)$. We shall prove $1\left(4^{\prime}\right)$. There are four cases: (i) $\alpha=0$, (ii) $0<\alpha<1 / 4$, (iii) $\alpha=$ $1 / 4$ and (iv) $1 / 4<\alpha<1 / 2$.

Case (i). The desired conclusion follows from Bessel's inequality.

To demonstrate the remaining cases we set

$$
\begin{gathered}
\Omega_{\mu}(x)=\sum_{2^{\mu-1}}^{2^{\mu}-1} a_{n} \omega_{n}(x), \quad W_{\mu}=\sum_{2^{\mu-1}}^{2^{\mu}-1}\left|a_{n}\right|^{2} n^{2 \alpha}, \\
I_{\nu \mu}=\int_{0}^{1}\left|\Omega_{\mu}(x) \Omega_{\nu}(x)\right| x^{-2 \alpha} d x .
\end{gathered}
$$

We further define

$$
I_{\nu \mu}^{(1)}=\int_{0}^{\varepsilon}\left|\Omega_{\mu}(x) \Omega_{\nu}(x)\right| x^{-2 \alpha} d x, \quad I_{\nu \mu}^{(2)}=\int_{\varepsilon}^{1}\left|\Omega_{\mu}(x) \Omega_{\nu}(x)\right| x^{-2 \alpha} d x .
$$

We begin by proving two inequalities we shall use repeatedly:

$$
\begin{aligned}
& \underset{0 \leqq x \leqq 1}{\operatorname{l.u}_{0} \text { b. }}\left|\Omega_{\mu}(x)\right| \leqq M \sum_{2^{\mu-1}}^{2^{\mu}-1}\left|a_{n}\right| \\
& \leqq M\left[\sum_{2}^{\mu-1}\left|a_{n}\right|^{2} n^{2 \alpha}\right]^{1 / 2}\left[\sum_{2^{\mu-1}}^{2^{\mu}-1} n^{-2 \alpha}\right]^{1 / 2} \\
& \leqq A W_{\mu}^{1 / 2} 2^{-\alpha \mu+\mu / 2} ; \\
& {\left[\int_{0}^{1}\left|\Omega_{\mu}(x)\right|^{2} d x\right]^{1 / 2}=\left[\sum_{2}^{\sum^{\mu-1}}\left|a_{n}\right|^{2}\right]^{1 / 2}} \\
& \leqq A W_{\mu}^{1 / 22^{-\alpha \mu}} .
\end{aligned}
$$

Here and later $A$ will be any constant depending only on $M$ and $\alpha$.

Case (ii). Suppose that $\nu \geqq \mu$. We have

$$
\begin{aligned}
I_{\nu \mu}^{(1)} & \leqq\left[\operatorname{liu}_{0 \leqq x \leqq \varepsilon}\left|\Omega_{\mu}(x)\right|\right]\left[\int_{0}^{\varepsilon} x^{-4 \alpha} d x\right]^{1 / 2}\left[\int_{0}^{\varepsilon}\left|\Omega_{\nu}(x)\right|^{2} d x\right]^{1 / 2} \\
& \leqq A \varepsilon^{1 / 2-2 \alpha} W_{\mu}^{1 / 2} W_{\nu}^{1 / 2} 2^{-\alpha \mu-\alpha \nu+\mu / 2}
\end{aligned}
$$

and

$$
\begin{aligned}
I_{\nu \mu}^{(2)} & \leqq\left[\operatorname{liu.b}_{\varepsilon \leqq x \leqq 1} x^{-2 \alpha}\right]\left[\int_{\varepsilon}^{1}\left|\Omega_{\mu}(x)\right|^{2} d x\right]^{1 / 2}\left[\int_{\varepsilon}^{1}\left|\Omega_{\nu}(x)\right|^{2} d x\right]^{1 / 2} \\
& \leqq A \varepsilon^{-2 \alpha} W_{\mu}^{1 / 2} W_{\nu}^{1 / 2} 2^{-\alpha \mu-\alpha \nu} .
\end{aligned}
$$


Setting $\varepsilon=2^{-\mu}$ we find that (for all $\mu$ and $\nu$ )

$$
I_{\nu \mu} \leqq A W_{\mu}^{1 / 2} W_{\nu}^{1 / 2} 2^{-\alpha|\nu-\mu|} .
$$

Since $f(x)=\sum_{\mu=0}^{\infty} \Omega_{\mu}(x)$ we have

$$
\begin{aligned}
\int_{0}^{1}|f(x)|^{2} x^{-2 \alpha} d x & \leqq \sum_{\mu, \nu=0}^{\infty} I_{\nu \mu} \leqq A \sum_{\mu, \nu=0}^{\infty} W_{\mu}^{1 / 2} W_{\nu}^{1 / 2} 2^{-\alpha|\nu-\mu|} \\
& \leqq A\left[\sum_{\mu=1}^{\infty} W_{\mu} \sum_{\nu=0}^{\infty} 2^{-\alpha|\nu-\mu|}\right]^{1 / 2}\left[\sum_{\nu=1}^{\infty} W_{\nu} \sum_{\mu=0}^{\infty} 2^{-\alpha|\nu-\mu|}\right]^{1 / 2} .
\end{aligned}
$$

We have

$$
\sum_{\mu=1}^{\infty} 2^{-\alpha|\nu-\mu|} \leqq A
$$

from which it follows that

$$
\int_{0}^{1}|f(x)|^{2} x^{-2 \alpha} d x \leqq A \sum_{n=1}^{\infty}\left|a_{n}\right|^{2} n^{2 \alpha} .
$$

Case (iii): $\alpha=1 / 4$. Suppose that $\nu \geqq \mu$. We have

$$
\begin{aligned}
& I_{\nu \mu}^{(1)} \leqq\left[\operatorname{liu}_{0 \leqq x \leqq \varepsilon}\left|\Omega_{\mu}(x)\right|\right]\left[\operatorname{liu}_{0 \leqq x \leqq \varepsilon}\left|\Omega_{\nu}(x)\right|\right] \int_{0}^{\varepsilon} x^{-1 / 2} d x \\
& \leqq A \varepsilon^{1 / 2} W_{\mu}^{1 / 2} W_{\nu}^{1 / 2} 2^{(\mu+\nu) / 4} ; \\
& I_{\nu \mu}^{(2)} \leqq\left[\int_{\varepsilon}^{1}\left|\Omega_{\nu}(x)\right|^{2} d x\right]^{1 / 2}\left[\int_{\varepsilon}^{1} x^{-1}\left|\Omega_{\mu}(x)\right|^{2} d x\right]^{1 / 2} \\
& \leqq\left[\int_{\varepsilon}^{1}\left|\Omega_{\gamma}(x)\right|^{2} d x\right]^{1 / 2}\left[\operatorname{liu}_{\varepsilon \leqq x \leqq 1}\left|\Omega_{\mu}(x)\right|\right]^{1 / 2}\left[\int_{\varepsilon}^{1} x^{-1}\left|\Omega_{\mu}(x)\right| d x\right]^{1 / 2} \\
& \leqq\left[\int_{\varepsilon}^{1}\left|\Omega_{\nu}(x)\right|^{2} d x\right]^{1 / 2}\left[\operatorname{lou}_{\varepsilon \leqq x \leqq 1}\left|\Omega_{\mu}(x)\right|\right]^{1 / 2}\left[\int_{\varepsilon}^{1}\left|\Omega_{\mu}(x)\right|^{2} d x\right]^{1 / 4}\left[\int_{\varepsilon}^{1} x^{-2} d x\right]^{1 / 4} \\
& \leqq A \varepsilon^{-1 / 4} W_{\mu}^{1 / 2} W_{\nu}^{1 / 2} 2^{-\nu / 4} \text {. }
\end{aligned}
$$

Choosing $\varepsilon=2^{-\mu / 3-2 \nu / 3}$ we obtain (for all $\mu$ and $\nu$ )

$$
I_{\nu \mu} \leqq A W_{\mu}^{1 / 2} W_{\nu}^{1 / 2} 2^{-|\nu-\mu| / 12},
$$

and the proof may be completed as before.

Case (iv): $1 / 4<\alpha<1 / 2$. We again suppose $\nu \geqq \mu$. We have

$$
\begin{aligned}
I_{\nu \mu}^{(1)} & \leqq\left[\operatorname{liu}_{0 \leqq x \leqq \varepsilon}\left|\Omega_{\mu}(x)\right|\right]\left[\operatorname{lou}_{0 \leqq x \leqq \varepsilon}\left|\Omega_{\nu}(x)\right|\right] \int_{0}^{\varepsilon} x^{-2 \alpha} d x \\
& \leqq A \varepsilon^{1-2 \alpha} W_{\mu}^{1 / 2} W_{\nu}^{1 / 2} 2^{-\alpha \mu-\alpha \nu+\mu / 2+\nu / 2} \\
I_{\nu \mu}^{(2)} & \leqq\left[\operatorname{liu}_{\varepsilon \leqq x \leqq 1}\left|\Omega_{\mu}(x)\right|\right]\left[\int_{\varepsilon}^{1} x^{-4 \alpha} d x\right]^{1 / 2}\left[\int_{\varepsilon}^{1}\left|\Omega_{\nu}(x)\right|^{2} d x\right]^{1 / 2}
\end{aligned}
$$




$$
\leqq A \varepsilon^{1 / 2-2 \alpha} W_{\mu}^{1 / 2} W_{\nu}^{1 / 2} 2^{-\alpha \mu-\alpha \nu+\mu / 2} .
$$

Choosing $\varepsilon=2^{-\nu}$ we find that (for all $\mu$ and $\nu$ )

$$
I_{\mu \nu} \leqq A W_{\mu}^{1 / 2} W_{\nu}^{1 / 2} 2^{(1 / 2-\alpha)|\nu-\mu|} .
$$

The proof of the inequality $1(4)$ is now complete.

It is evident that $1\left(4^{\prime}\right)$ remains valid if the condition $\sum_{1}^{\infty}\left|a_{n}\right|<\infty$ is abandoned, provided that $f(x)$ is interpreted as limit in the mean.

Let $a_{1}, a_{2}, \ldots$ be a sequence of complex constants which approach 0 as $n$ approaches $\infty$. We denote by $a_{1}^{*}, a_{2}^{*}, \ldots$ the sequence $\left|a_{1}\right|$, $\left|a_{2}\right|, \ldots$ arranged in non-increasing order. Let $f(x)$ be a complex valued measurable function defined on $[0,1]$. We denote by $f^{*}(x)$ the function equimeasurable with $|f(x)|$ and non-increasing. A simple and well-known argument, see [2; pp. 207-211], enables us to restate our inequalities in the stronger form,

$$
\begin{aligned}
\int_{0}^{1}\left[f^{*}(x)\right]^{2} x^{-2 \alpha} d x \leqq A(\alpha) \sum_{n=1}^{\infty}\left[\alpha_{n}^{*}\right]^{2} n^{2 \alpha} & (0 \leqq \alpha<1 / 2) ; \\
\sum_{n=1}^{\infty}\left[a_{n}^{*}\right]^{2} n^{-2 \alpha} \leqq A(\alpha) \int_{0}^{1}\left[f^{*}(x)\right]^{2} x^{-2 \alpha} d x . & (0 \leqq \alpha<1 / 2) .
\end{aligned}
$$

3. We now deduce the first of Riesz's inequalities. Let $b_{1}, b_{2} \ldots$ be given such that $B=\left(\sum_{1}^{\infty}\left|b_{n}\right|^{p}\right)^{1 / p}$ is finite where $1<p \leqq 2$. We may write $B^{p}=\sum_{1}^{\infty}\left[b_{n}^{*}\right]^{p}$.

Since $b_{n}^{*}$ is non-increasing $n\left[b_{n}^{*}\right]^{p} \leqq B^{p}$ or $b_{n}^{*} \leqq n^{-1 / p} B$. It follows that

$$
\sum_{n=1}^{\infty}\left[b_{n}^{*}\right]^{2} n^{(2-p) / p} \leqq B^{2-p} \sum_{n=1}^{\infty}\left[b_{n}^{*}\right]^{p}=B^{2} .
$$

By $2\left(3^{\prime}\right)$ we have, if $f(x)=\sum_{n=1}^{\infty} b_{n} \omega_{n}(x)$,

$$
\int_{0}^{1}\left[\left(f^{*}(x)\right]^{2} x^{-(2-p) / p} d x \leqq\left[A\left(\frac{2-p}{2 p}\right)\right]^{2} B^{2} .\right.
$$

Let $F=\left[\int_{0}^{1}|f(x)|^{q} d x\right]^{1 / q}$ where $p^{-1}+q^{-1}=1$. We have $F^{q}=\int_{0}^{1}\left[f^{*}(x)\right]^{q} d x$.

Since $f^{*}(x)$ is non-increasing $x\left[f^{*}(x)\right]^{\alpha} \leqq F^{q}$ or $f^{*}(x) \leqq x^{-1 / q} F$. It follows that

$$
F^{2}=F^{2-q} \int_{0}^{1}\left[f^{*}(x)\right]^{q} d x \leqq \int_{0}^{1}\left[f^{*}(x)\right]^{2} x^{-(q-2) / q} d x .
$$

Since $(q-2) / q=(2-p) / p$ we obtain 


$$
\left[\int_{0}^{1}|f(x)|^{q} d x\right]^{1 / q} \leqq B(p)\left[\sum_{1}^{\infty}\left|b_{n}\right|^{p}\right]^{1 / p}
$$

where $B(p)$ may be taken as $\left[A\left(\frac{2-p}{2 p}\right)\right]$. A similar argument serves to establish the other Riesz inequality.

4. It is natural to conjecture the existence of a general inequality which includes Paley's inequalities 1(3) and the inequalities 1(4). We shall prove that

$$
\int_{0}^{1}|f(x)|^{r} x^{-r \gamma} d x \leqq A_{1}^{\prime \prime}(r, \gamma) \sum_{n=1}^{\infty}\left|a_{n}\right|^{\mid r} n^{r-2+r \gamma} \quad(2 \leqq r<\infty, 0 \leqq \gamma<1 / r),
$$

$$
\sum_{n=1}^{\infty}\left|a_{n}\right|^{r} n^{-r \gamma} \leqq A_{2}^{\prime \prime}(r, \gamma) \int_{0}^{1}|f(x)|^{r} x^{r-2+\gamma \gamma} d x \quad(2 \leqq r<\infty, 0 \leqq \gamma<1 / r),
$$

$$
\int_{0}^{1}|f(x)|^{r} x^{r-2-r \gamma} d x \leqq A_{3}^{\prime \prime}(r, \gamma) \sum_{n=1}^{\infty}\left|a_{n}\right|^{r} n^{r \gamma} \quad(1<r \leqq 2,0 \leqq \gamma<1-1 / r) \text {, }
$$

(4) $\sum_{n=1}^{\infty}\left|a_{n}\right|^{r} n^{r-2-r \gamma} \leqq A_{4}^{\prime \prime}(r, \gamma) \int_{0}^{1}|f(x)|^{r} x^{r \gamma} d x \quad(1<r \leqq 2,0 \leqq \gamma<1-1 / r)$.

Let us prove (1). Choose $q, 2<r<q$. We have

$$
\begin{aligned}
& {\left[\int_{0}^{1}|f(x)|^{2} x^{-2 \alpha} d x\right]^{1 / 2} \leqq A(\alpha)\left[\sum_{n=1}^{\infty}\left|a_{n}\right|^{2} n^{2 \alpha}\right]^{1 / 2},} \\
& {\left[\int_{0}^{1}|f(x)|^{q} d x\right]^{1 / 2} \leqq A_{1}^{\prime}(q)\left[\sum_{n=1}^{\infty}\left|a_{n}\right|^{q} n^{q-2}\right]^{1 / q} .}
\end{aligned}
$$

We write (formally)

$$
|g(x)|^{2} M(x)=|f(x)|^{2} x^{-2 \alpha}, \quad|g(x)|^{q} M(x)=|f(x)|^{q} .
$$

These relations suggest that we define $g(x)$ and $M(x)$ by

$$
g(x)=f(x) x^{2 \alpha /(q-2)}, \quad M(x)=x^{-2 \alpha q /(q-2)} .
$$

Similarly from the (formal) relations

$$
\left|b_{n}\right|^{2} m(n)=\left|a_{n}\right|^{2} n^{2 \alpha}, \quad\left|b_{n}\right|^{q} m(n)=\left|a_{n}\right|^{q} n^{q-2},
$$

we are lead to the definitions

$$
b_{n}=a_{n} n^{1-2 \alpha /(q-2)}, \quad m(n)=n^{2(q \alpha-q+2) /(q-2)} .
$$

The mapping $T\left\{b_{n}\right\}_{1}^{\infty}=g(x)$ is a linear transformation, and we have

$$
\begin{aligned}
& \int_{0}^{1}|g(x)|^{2} M(x) d x \leqq A(\alpha) \sum_{n=1}^{\infty}\left|b_{n}\right|^{2} m(n), \\
& \int_{0}^{1}|g(x)|^{q} M(x) d x \leqq A^{\prime}(q) \sum_{n=1}^{\infty}\left|b_{n}\right|^{q} m(n) .
\end{aligned}
$$


By the Riesz interpolation theorem,

$$
\left[\int_{0}^{1}|g(x)|^{r} M(x) d x\right]^{1 / r} \leqq C(\alpha, r, q)\left[\sum_{n=1}^{\infty}\left|b_{n}\right|^{r} m(n)\right]^{1 / r} .
$$

Now

$$
\begin{gathered}
\int_{0}^{1}|g(x)|^{r} M(x) d x=\int_{0}^{1}|f(x)|^{r} x^{2 \alpha r /(q-2)} x^{-2 \alpha q /(q-2)} d x, \\
\sum_{n=1}^{\infty}\left|b_{n}\right|^{r} m(n)=\sum_{n=1}^{\infty}\left|a_{n}\right|^{r} n^{r} n^{-2 \alpha r /(q-2)} n^{2(q \alpha-q+2) /(q-2)} .
\end{gathered}
$$

If $\gamma$ is defined by the equation

$$
r \gamma=-\frac{2 \alpha(r-q)}{q-2}
$$

then (5) can be rewritten as

$$
\int_{0}^{1}|f(x)|^{r} x^{-r \gamma} d x \leqq C(\alpha, r, q) \sum_{n=1}^{\infty}\left|a_{n}\right|^{r} n^{r-2+r \gamma} .
$$

It is evident from (6) that, by properly choosing $\alpha$ and $q$, $\gamma$ can assume any value in the range $0 \leqq r<1 / r$. Thus we have established (1). The relations (2), (3) and (4) can be dealt with similarly. For the special case of Fourier series these inequalities have been established by H. R. Pitt [3].

The "*" forms of these inequalities are also true.

5. In the present section we shall prove a result which is a slight variant of the Riesz-Thorin convexity theorem. While this is probably known I have not been able to find a reference for it.

Let $\left[T_{i j}\right](i=1, \cdots, m ; j=1, \cdots, n)$ be a complex matrix, and let

$$
a_{i}=\sum_{j=1}^{n} T_{i j} b_{j} \quad(i=1, \cdots, m) .
$$

Let $\mu_{i}, \sigma_{i}$ be positive for $i=1, \cdots, m$ and $\nu_{j}, \tau_{j}$ be positive for $j=1$, $\cdots, n$. For $1 \leqq p, q \leqq \infty$ let

$$
A(\alpha, \beta)=\text { l.u.b. }\left[\sum_{i=1}^{m}\left|a_{i}\right|^{p} \mu_{i}^{p \alpha} \sigma_{i}\right]^{1 / p},
$$

where the least upper bound is extended over all sets $\left(b_{1}, \cdots, b_{n}\right)$ such that

$$
\left[\sum_{j=1}^{n}\left|b_{j}\right|_{\nu_{j}^{q \beta}} \tau_{j}\right]^{1 / q}=1
$$

We assert that $\log A(\alpha, \beta)$ is convex for $-\infty<\alpha, \beta<\infty$; that is, if 


$$
\alpha=(1-\theta) \alpha_{1}+\theta \alpha_{2}, \quad \beta=(1-\theta) \beta_{1}+\theta \beta_{2} \quad(0<\theta<1),
$$

then

$$
A(\alpha, \beta) \leqq A\left(\alpha_{1}, \beta_{1}\right)^{1-\theta} A\left(\alpha_{2}, \beta_{2}\right)^{\theta} .
$$

To prove this let $\left(b_{1}, \cdots, b_{n}\right)$ be fixed, such that $(2)$ is satisfied and let $\left(c_{1}, \cdots, c_{m}\right)$ be such that

$$
\left[\sum_{i=1}^{m}\left|c_{i}\right|^{p^{\prime}} \mu_{i}^{-p^{\prime} \alpha} \sigma_{i}\right]^{1 / p^{\prime}}=1, \quad 1 / p+1 / p^{\prime}=1
$$

Consider

$$
\boldsymbol{f}(w)=\sum_{i=1}^{m}\left[\sum_{j=1}^{n} T_{i j} b_{j} \nu_{j}^{\beta-\beta_{1}+w\left(\beta_{1}-\beta_{2}\right)}\right] c_{i} \mu_{i}^{-\alpha+\alpha_{1}+w\left(-\alpha_{1}+\alpha_{2}\right)} \sigma_{i} .
$$

The function $\boldsymbol{f}(w)$ is entire and is bounded in every vertical strip. Let us set

$$
\begin{gathered}
b_{j}(w)=b_{j} \nu_{j}^{\beta-\beta_{1}+w\left(\beta_{1}-\beta_{2}\right)}, \quad c_{i}(w)=c_{i} \mu_{i}^{-\alpha+\alpha_{1}+w\left(-\alpha_{1}+\alpha_{2}\right)}, \\
a_{i}(w)=\sum_{j=1}^{n} T_{i j} b_{j}(w),
\end{gathered}
$$

so that

$$
f(w)=\sum_{i=1}^{m} a_{i}(w) c_{i}(w) \sigma_{i}
$$

We have

$$
\left[\sum_{j=1}^{n}\left|b_{j}(i v)\right|^{q}{ }_{j}^{q \beta} \tau_{1} \tau_{j}\right]^{1 / \alpha}=1
$$

and thus

$$
\left[\sum_{i=1}^{m}\left|a_{\imath}(i v)\right|^{p} \mu_{i}^{p \alpha_{1}} \sigma_{i}\right]^{1 / p} \leqq A\left(\alpha_{1}, \beta_{1}\right)
$$

further

$$
\left[\sum_{i=1}^{m}\left|c_{i}(i v)\right|^{p^{\prime}} \mu_{i}^{-p^{\prime} \alpha_{1} \sigma_{i}}\right]^{1 / p^{\prime}}=1 .
$$

Applying Hölder's inequality we obtain

$$
|\boldsymbol{f}(i v)| \leqq A\left(\alpha_{1}, \beta_{1}\right) .
$$

We may similarly show that

$$
|f(1+i v)| \leqq A\left(\alpha_{2}, \beta_{2}\right) .
$$

By the three lines theorem 


$$
|\mathbf{f}(\theta)| \leqq A\left(\alpha_{1}, \beta_{1}\right)^{1-\theta} A\left(\alpha_{2}, \beta_{2}\right)^{\theta}
$$

which is equivalent to the inequality

$$
\left|\sum_{i=1}^{m} a_{i} c_{i} \sigma_{i}\right| \leqq A\left(\alpha_{1}, \beta_{1}\right)^{1-\theta} A\left(\alpha_{2}, \beta_{2}\right)^{\theta} .
$$

Since this holds for all $\left(c_{1}, c_{2}, \cdots, c_{m}\right)$ satisfying (5) this implies that

$$
\left[\sum_{i=1}^{m}\left|a_{i}\right|^{p} \mu_{i}^{p \alpha} \sigma_{i}\right]^{1 / p} \leqq A\left(\alpha_{1}, \beta_{1}\right)^{1-\theta} A\left(\alpha_{2}, \beta_{2}\right)^{\theta}
$$

thus verifying our assertion. We have tacitly assumed above that $p$, $q<\infty$. The case where $p$ or $q$ or both are $\infty$ cae be dealt with by passing to the limit.

We shall now apply this to show that if $A(\alpha)$ is defined as in $\S 2$ then $\log A(\alpha)$ is convex. Let

$$
T_{i j}=\int_{(j-1) / n}^{j / n} \omega_{i}(x) d x \quad(i=1, \cdots, m ; j=1, \cdots, n)
$$

and let $f(x)$ be a step function taking the value $b_{j}$ for $(j-1) / n \leqq x<$ $j / n$. If $a_{i}=\int_{0}^{1} f(x) \omega_{i}(x) d x$ then

$$
a_{i}=\sum_{j=1}^{m} T_{i j} b_{j}
$$

Let

$$
A_{m, n}(\alpha)=\text { l.u.b. }\left[\sum_{i=1}^{m}\left|a_{i}\right|^{2} i^{-2 \alpha}\right]^{1 / 2}
$$

the least upper bound being taken over all $b_{1}, \cdots, b_{n}$ such that

$$
\left[\sum_{j=1}^{n}\left|b_{j}\right|^{2} n^{-1}(j / n)^{-2 \alpha}\right]^{1 / 2}=1
$$

For every $m$ and $n, A_{m, n}$ is a logarithmically convex function of $\alpha$. We have

$$
\lim _{m, n \rightarrow \infty} A_{m, n}(\alpha)=A(\alpha)
$$

and from this if follows that $A(\alpha)$ is logarithmically convex.

Because of this fact it is sufficient in $\S 2$ to deal only with cases (i) and (iv), since (ii) and (iii) then follow by interpolation.

\section{REFERENCES}

1. R. E. A. C. Paley, Some theorems on orthogonal functions, Studia Math., 3 (1931), 226-239. 
2. A. Zygmund, Trigonometrical series, Lwow 1935.

3. H. R. Pitt, Theorems on Fourier series and power series, Duke Math. J., 3 (1937), 747-755.

WASHINGTON UNIVERSITY 


\section{PACIFIC JOURNAL OF MATHEMATICS}

\section{EDITORS}

H. L. Royden

Stanford University

Stanford, California

E. HewitT

University of Washington

Seattle 5 , Washington
R. P. Dilworth

California Institute of Technology Pasadena 4, California

E. G. Straus

University of California

Los Angeles 24, California

\section{ASSOCIATE EDITORS}

E. F. BECKENBACH

C. E. BURGESS

H. BUSEMANN

H. FEDERER

\author{
M. HALL \\ P. R. HALMOS \\ V. GANAPATHY IYER \\ R. D. JAMES
}

M. S. KNEBELMAN

I. NIVEN

T. G. OSTROM

M. M. SCHIFFER
J. J. STOKER

G. SZEKERES

F. WOLF

K. YOSIDA

\section{SUPPORTING INSTITUTIONS}

UNIVERSITY OF BRITISH COLUMBIA

CALIFORNIA INSTITUTE OF TECHNOLOGY

UNIVERSITY OF CALIFORNIA

MONTANA STATE UNIVERSITY

UNIVERSITY OF NEVADA

OREGON STATE COLLEGE

UNIVERSITY OF OREGON

UNIVERSITY OF SOUTHERN CALIFORNIA
STANFORD UNIVERSITY

UNIVERSITY OF UTAH

WASHINGTON STATE COLLEGE

UNIVERSITY OF WASHINGTON

AMERICAN MATHEMATICAL SOCIETY CALIFORNIA RESEARCH CORPORATION HUGHES AIRCRAFT COMPANY 


\section{Pacific Journal of Mathematics}

\section{Vol. 6, No. $1 \quad$ November, 1956}

David Blackwell, An analog of the minimax theorem for vector payoffs..... 1

L. W. Cohen, A non-archimedian measure in the space of real

sequences ..................................... 9

George Bernard Dantzig, Constructive proof of the Min-Max theorem ..... 25

Jim Douglas, On the numerical integration of quasilinear parabolic

differential equations ............................... 35

James Michael Gardner Fell, A note on abstract measure ............. 43

Isidore Isaac Hirschman, Jr., A note on orthogonal systems . . . . . . . . . . 47

Frank Harary, On the number of dissimilar line-subgraphs of a given

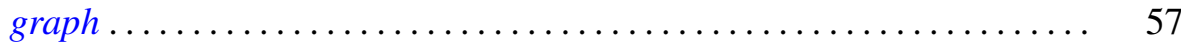

Newton Seymour Hawley, Complex bundles with Abelian group......... 65

Alan Jerome Hoffman, Morris Newman, Ernst Gabor Straus and Olga

Taussky, On the number of absolute points of a correlation ...........

Ernst Gabor Straus and Olga Taussky, Remark on the preceding paper.

Algebraic equations satisfied by roots of natural numbers . . ........ 97

Ralph D. James, Summable trigonometric series ................. 99

Gerald R. Mac Lane, Limits of rational functions . . . . . . . . . . . . . . . 111

F. Oberhettinger, Note on the Lerch zeta function ................. 117

Gerald C. Preston, On locally compact totally disconnected Abelian groups and their character groups ........................... 121

Vikramaditya Singh and W. J. Thron, On the number of singular points, located on the unit circle, of certain functions represented by

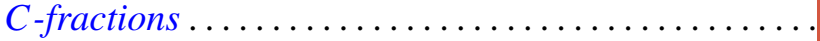

Sherman K. Stein, The symmetry function in a convex body ... 145 Edwin Weiss, Boundedness in topological rings.............

Albert Leon Whiteman, A sum connected with the series for the partition

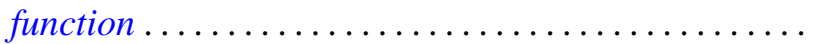

Alfred B. Willcox, Some structure theorems for a class of Banach algebras.

Joseph Lawrence Zemmer, Some remarks on p-rings and their Boolean geometry... 